\title{
Workload of diagnostic radiologists in the foreseeable future based on recent scientific advances: growth expectations and role of artificial intelligence
}

Thomas C. Kwee ${ }^{1 *}$ (ID and Robert M. Kwee

\begin{abstract}
Objective: To determine the anticipated contribution of recently published medical imaging literature, including artificial intelligence (Al), on the workload of diagnostic radiologists.

Methods: This study included a random sample of 440 medical imaging studies published in 2019. The direct contribution of each study to patient care and its effect on the workload of diagnostic radiologists (i.e., number of examinations performed per time unit) was assessed. Separate analyses were done for an academic tertiary care center and a non-academic general teaching hospital.
\end{abstract}

Results: In the academic tertiary care center setting, $65.0 \%$ (286/440) of studies could directly contribute to patient care, of which 48.3\% (138/286) would increase workload, 46.2\% (132/286) would not change workload, 4.5\% (13/286) would decrease workload, and 1.0\% (3/286) had an unclear effect on workload. In the non-academic general teaching hospital setting, $63.0 \%$ (277/240) of studies could directly contribute to patient care, of which $48.7 \%(135 / 277)$ would increase workload, 46.2\% (128/277) would not change workload, 4.3\% (12/277) would decrease workload, and 0.7\% $(2 / 277)$ had an unclear effect on workload. Studies with Al as primary research area were significantly associated with an increased workload $(p<0.001)$, with an odds ratio (OR) of 10.64 (95\% confidence interval $(C \mathrm{Cl}) 3.25-34.80)$ in the academic tertiary care center setting and an OR of 10.45 (95\% Cl 3.19-34.21) in the non-academic general teaching hospital setting.

Conclusions: Recently published medical imaging studies often add value to radiological patient care. However, they likely increase the overall workload of diagnostic radiologists, and this particularly applies to Al studies.

Keywords: Artificial intelligence, Radiology, Radiologists, Research, Workload

\footnotetext{
*Correspondence: thomaskwee@gmail.com

${ }^{1}$ Medical Imaging Center, Departments of Radiology, Nuclear Medicine and Molecular Imaging, University Medical Center Groningen, University of Groningen, Hanzeplein 1, P.O. Box 30.001, 9700 RB Groningen, The Netherlands

Full list of author information is available at the end of the article
}

\section{Key points}

- In an academic tertiary care center, $65.0 \%$ of recently published medical imaging studies could directly contribute to patient care, of which $48.3 \%$ would increase and $4.5 \%$ would decrease workload.

- In a non-academic general teaching hospital, $63.0 \%$ of recently published medical imaging studies could directly contribute to patient care, of which $48.7 \%$ would increase and $4.3 \%$ would decrease workload. 
- Recently published medical imaging studies with AI as primary research area are significantly associated with an increased workload.

\section{Introduction}

The workload of radiologists (i.e., the product of the number and complexity of examinations performed per time unit) has increased considerably over the past decades. This is largely due to the growth in the number of cross-sectional imaging examinations (particularly CT and MRI), increased complexity of image interpretation because of the acquisition of larger datasets, and declining imaging reimbursements [1-4]. The latter forces radiology practices to increase productivity to maintain income levels, while limiting their financial possibilities to employ new staff. Consequently, the overall workload per radiologist has considerably increased over the past years. Not surprisingly, burnout is recognized as an increasingly important problem among radiologists [5, 6]. Work overload may also compromise the quality and safety of patient care that can be provided by radiologists [7-9].

There are many factors that influence the utilization of medical imaging [10]. Scientific evidence can be considered as perhaps the most important driver, because research-based advances shape the way how radiology practice is performed in the future. Currently, however, it is unclear how recently published literature contributes to the workload in the field of radiology. This information would be important to radiologists and other stakeholders (including healthcare systems and governmental bodies) who aim to maintain a radiology workforce that is sufficiently large to meet clinical demands at high standards.

Meanwhile, there are also voices that speculate that artificial intelligence (AI) is expected to speed up scan time, make more accurate diagnoses, and ease the workload of radiologists [11]. Although the speculation that AI will decrease workload has not yet been supported by evidence, it can already have an important impact on political and strategic decisions. Based on this speculation, policy makers may already decide not to increase or even to limit the number of residents that can be enrolled into radiology training programs, may limit financial resources to employ (new) radiologists, and may further cut reimbursements for imaging procedures. This may have detrimental effects that cannot be rapidly reversed when the workload of radiologists actually proves to keep on increasing. Interestingly, in a recent survey among European Society of Radiology (ESR) members, 74.7\% (504/675) expected an impact of AI on total reporting workload, with $50.8 \%(256 / 504)$ of them expecting a reduced reporting workload and 49.2\% (248/504) expecting the opposite scenario [12]. Since the effect of AI on future workload remains unclear, and associated practical consequences are very relevant, it is crucial to fill in this knowledge gap. It is hypothesized that most recent scientific evidence increases workload in radiology practice, while there is no net effect of AI on radiologists' workload based on the recent ESR survey results [12].

The purpose of this study was therefore to determine the anticipated contribution of recently published medical imaging literature on the workload of diagnostic radiologists.

\section{Materials and methods Study design}

This study used literature data and did not require ethical review board approval or patient consent. Studies that were published in any of the 20 highly ranked clinical imaging journals and 20 highly ranked general medicine and clinical specialty journals (according to impact factor in the Journal Citation reports [13], that are listed in Table 1, were considered for inclusion in this study. All studies that were published in these 40 clinical journals in 2019 were retrieved from PubMed/Medline using the search term that is displayed in Additional file 1: Appendix 1 . Using the random number generator in Microsoft Excel (Microsoft Corporation), a random sample of 1,000 studies was drawn from the studies that were retrieved by the PubMed/Medline search. These 1,000 studies were reviewed for eligibility by consensus of two radiologists (T.C.K. \& R.M.K.). Studies were included if they concerned diagnostic imaging studies. Only original studies, systematic reviews and meta-analyses, were included. Case reports, conference proceedings, editorials, errata, guidelines/society statements, studies dealing with interventional radiology, letters to the editor, meeting summaries, non-systematic reviews, and studies clearly not related to medical imaging, were excluded. Studies dealing with imaging procedures that are not performed by radiologists in the authors' institutions, such as cardiac ultrasonography or endoscopic ultrasonography, were also excluded. Studies were excluded if clearly ineligible based on title and abstract; otherwise, the full text version was evaluated.

\section{Study analysis}

One radiologist affiliated to an academic tertiary care center in the north of the Netherlands (T.C.K., radiologist 1 ), and another radiologist affiliated to a non-academic general teaching hospital in the south of the Netherlands (R.M.K., radiologist 2), independently reviewed the full text of each included study. Radiologist 1 had 5 years of post-residency clinical radiology experience 
Table 1 Overview of 20 clinical imaging journals and 20 general medicine and clinical specialty journals whose studies were potentially eligible for inclusion

\begin{tabular}{ll}
\hline Imaging journals & General medicine and clinical specialty journals \\
\hline JACC: Cardiovascular Imaging & New England Journal of Medicine \\
Radiology & Lancet \\
Journal of Nuclear Medicine & Journal of the American Medical Association \\
European Journal of Nuclear Medicine and Molecular Imaging & Lancet Oncology \\
Clinical Nuclear Medicine & Journal of Clinical Oncology \\
Journal of Cardiovascular Magnetic Resonance & BMJ \\
Investigative Radiology & Lancet Neurology \\
European Heart Journal_Cardiovascular Imaging & Lancet Diabetes \& Endocrinology \\
Journal of the American College of Radiology & Lancet Respiratory Medicine \\
European Radiology & JAMA Oncology \\
Journal of Magnetic Resonance Imaging & Lancet Infectious Diseases \\
Insights into Imaging & Circulation \\
American Journal of Neuroradiology & European Heart Journal \\
Journal of Nuclear Cardiology & Annals of Internal Medicine \\
Quantitative Imaging in Medicine and Surgery & Journal of the American College of Cardiology \\
Clinical Neuroradiology & Gut \\
Korean Journal of Radiology & European Urology \\
Journal of Vascular and Interventional Radiology & JAMA Internal Medicine \\
American Journal of Roentgenology & Annals of Oncology \\
European Journal of Radiology & Blood
\end{tabular}

and radiologist 2 had 6 years of post-residency clinical radiology experience, and both radiologists had 15 years of experience in critically appraising scientific literature in radiology. The radiology departments in both institutions, which are among the largest in The Netherlands, are regularly audited by the Radiological Society of the Netherlands to fulfill quality standards of radiological care [14]. Both radiologists assessed whether the results of each individual study could directly contribute to patient care in their radiology practice (i.e., whether the results may be directly implemented in practice for the purpose of enhancing patient care). If this was the case, this contribution was then categorized as concerning:

(1) a completely new imaging application in their radiology practice.

(2) another type of imaging as an alternative for an existing imaging application in their radiology practice.

(3) an elaboration of on an existing imaging application in their radiology practice.

For contributions in category (2), it was qualitatively determined if the acquisition, post-processing, and interpretation times of the alternative type of imaging would be shorter than, as long as, or longer than that of the existing imaging application, or whether this remained unclear. Similarly, for contributions in category (3), it was qualitatively determined if the acquisition, postprocessing, and interpretation times of the existing imaging application would decrease, remain stable, increase, or whether this remained unclear. Note that acquisition time refers to the time required to obtain images of the human body with a certain imaging modality, post-processing time refers to the time required for the manipulation of medical images to derive additional qualitative or quantitative data [15], and interpretation time refers to the time required to analyze and interpret the acquired and post-processed imaging data. All studies in category (1) and any study in categories (2) or (3) with a net increase in acquisition, post-processing, and/or interpretation time were considered to increase workload. Any study in categories (2) or (3) with a net decrease in acquisition, post-processing, and/or interpretation time was considered to decrease workload. Any study in categories (2) or (3) without a net change in acquisition, post-processing, and/or interpretation time was considered to have no effect on workload. All other studies were considered to have an unclear impact on workload. Study examples are shown in Tables 2 and 3 [16-26].

\section{Statistical analysis}

Separate analyses were done for the previously mentioned academic tertiary care center and non-academic 


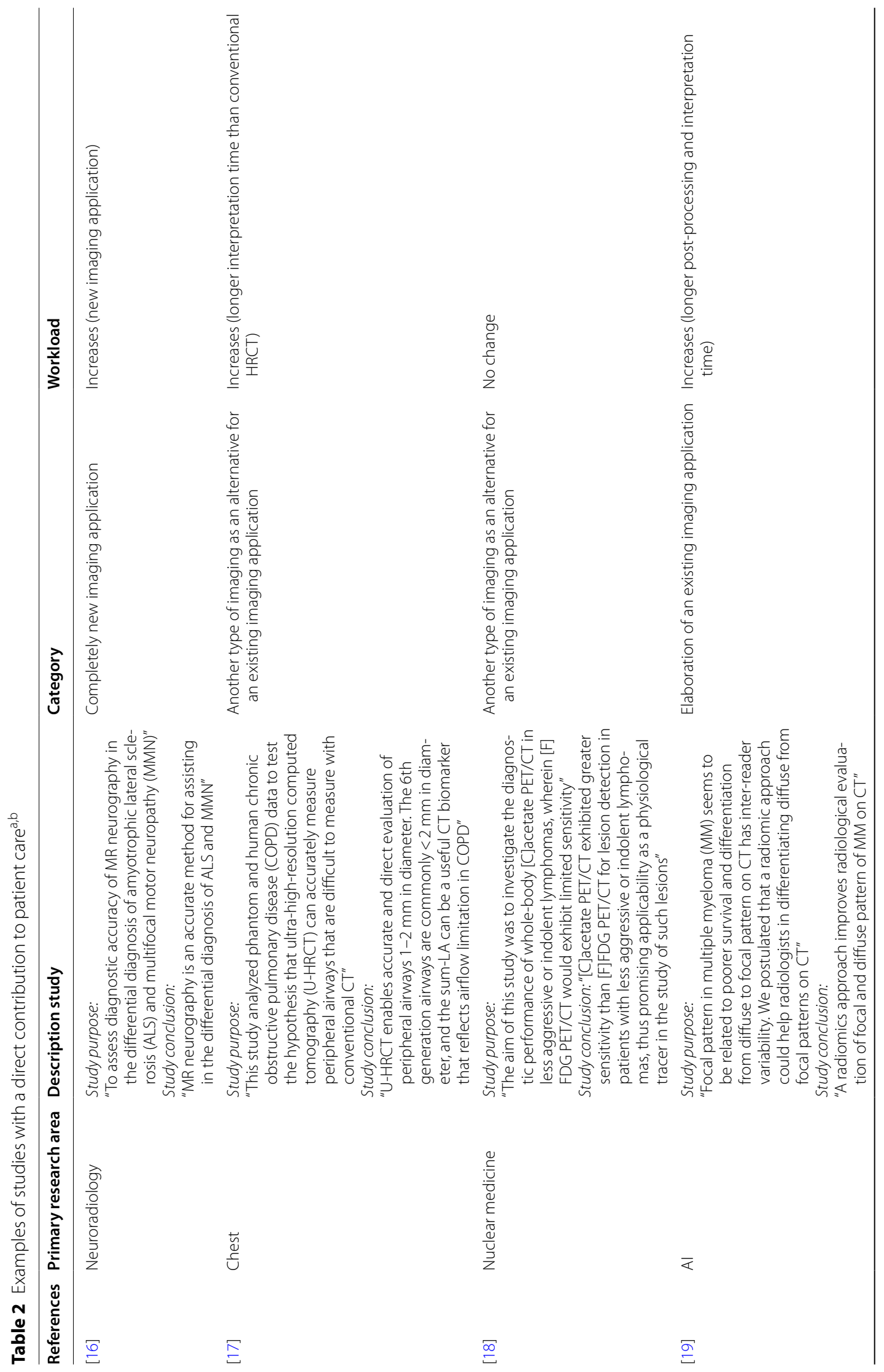




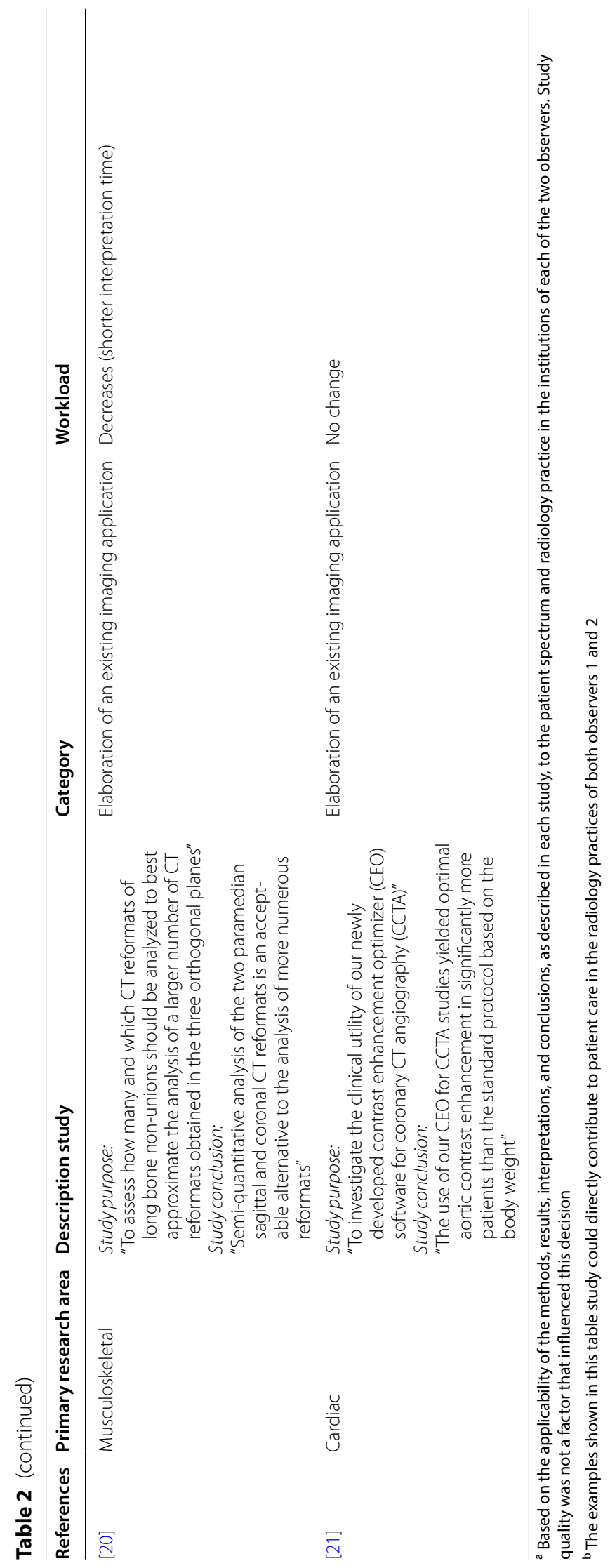


Table 3 Examples of studies without a direct contribution to patient care ${ }^{a, b}$

\begin{tabular}{|c|c|c|}
\hline References & Primary research area & Description study \\
\hline [22] & Nuclear medicine & $\begin{array}{l}\text { Study purpose: } \\
\text { "The aim of this study is to measure acute changes in NaF uptake in human bone due to exercise-induced } \\
\text { loading" } \\
\text { Study conclusion: } \\
\text { "Bone loading induces an acute response in bone physiology as quantified by [18F]NaF PET kinetics. } \\
\text { Dynamic imaging after bone loading using [18F]NaF PET is a promising diagnostic tool in bone physiol- } \\
\text { ogy and imaging of biomechanics" }\end{array}$ \\
\hline [23] & Magnetic resonance & $\begin{array}{l}\text { Study purpose: } \\
\text { "To qualitatively and quantitatively compare the image quality between single-shot echo-planar (SS-EPI) } \\
\text { and multi-shot echo-planar (IMS-EPI) diffusion-weighted imaging (DWI) in female pelvis" } \\
\text { Study conclusion: } \\
\text { "IMS-EPI showed better image quality with lower geometric distortion without affecting the quantification } \\
\text { of apparent diffusion coefficient, though the signal-to-noise ratio and contrast-to-noise ratio decreased } \\
\text { due to post-processing limitations" }\end{array}$ \\
\hline [24] & Breast & $\begin{array}{l}\text { Study purpose: } \\
\text { "To develop a fast three-dimensional method for simultaneous T1 and T2 quantification for breast imaging } \\
\text { by using MR fingerprinting" } \\
\text { Study conclusion: } \\
\text { "A method was developed for breast imaging by using the MR fingerprinting technique, which allows } \\
\text { simultaneous and volumetric quantification of T1 and T2 relaxation times for breast tissues" }\end{array}$ \\
\hline [25] & Gastrointestinal-abdominal & $\begin{array}{l}\text { Study purpose: } \\
\text { "To compare patient acceptability and burden of magnetic resonance enterography (MRE) and ultrasound } \\
\text { (US) to each other, and to other enteric investigations, particularly colonoscopy." } \\
\text { Study conclusion: } \\
\text { "MRE and US are well tolerated. Although MRE generates greater burden, longer recovery and is less } \\
\text { preferred than US, it is more acceptable than colonoscopy. Patients, however, place greater emphasis on } \\
\text { diagnostic accuracy than burden" }\end{array}$ \\
\hline [26] & Urogenital & $\begin{array}{l}\text { Study purpose: } \\
\text { "The objectives of this study were to assess whether the accuracy of urologists in identifying the presence } \\
\text { of clinically significant cancer based on a standardized multiparametric MRI set could be improved by } \\
\text { completion of a 2-d training course" } \\
\text { Study conclusion: } \\
\text { "Whilst we require expert radiologists to report prostate MRI, this study has demonstrated that identifica- } \\
\text { tion of clinically significant cancer on prostate MRI by urologists is improved following exposure to a 2-d } \\
\text { teaching course. These results would support efforts to integrate prostate MRI teaching courses into the } \\
\text { training of urologists managing patients with prostate cancer" }\end{array}$ \\
\hline
\end{tabular}

a Based on the applicability of the methods, results, interpretations, and conclusions, as described in each study, to the patient spectrum and radiology practice in the institutions of each of the two observers. Study quality was not a factor that influenced this decision

${ }^{b}$ The examples shown in this table study could not directly contribute to patient care in the radiology practices of both observers 1 and 2

general teaching hospital settings. The proportions of studies that could directly contribute to patient care, and those concerning (1) a completely new imaging application, (2) another type of imaging as an alternative for an existing imaging application, and (3) an elaboration of on an existing imaging application were calculated. Subsequently, the proportions of studies that would increase workload, decrease workload, have no effect on workload, and with an unclear impact on workload were calculated. Logistic regression analyses were performed to determine the association of increased workload with a study's primary research area (AI, breast, cardiac, chest, computed tomography, contrast media, emergency, experimental, gastrointestinal-abdominal, head-neck, magnetic resonance, multisystem, musculoskeletal, neuroradiology, nuclear medicine, oncology, pediatric, ultrasonography, urogenital, or vascular) and impact factor of the journal in which the study was published (according to the 2019 Journal Citation reports (13)). Studies that would increase workload were coded as " 1 " and studies that would not change or decrease workload were calculated as " 0 " for this purpose. The largest category was used as reference for the variable "study's research area." $p$ values less than 0.05 were considered statistically significant. Statistical analyses were executed using MedCalc version 17.2 Software (MedCalc).

\section{Results}

\section{Eligible studies}

Of the 1000 studies that were evaluated, 560 studies were excluded (Fig. 1). Finally, 440 studies remained for inclusion (Additional file 1: Appendix 2).

\section{Academic tertiary care center setting}

$65.9 \%(286 / 440)$ of studies could directly contribute to patient care, of which $84.6 \%(242 / 286)$ concerned an 


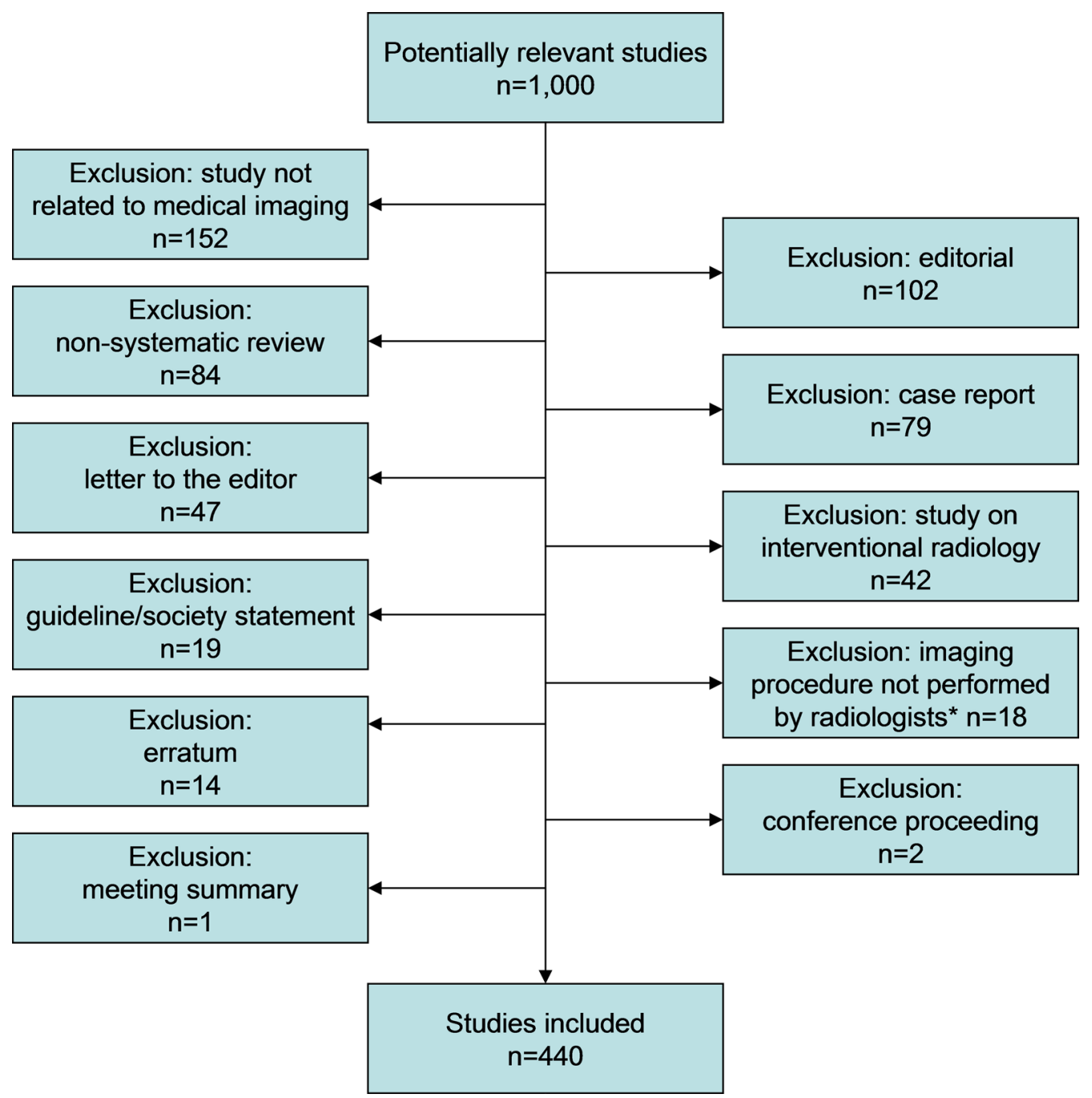

Fig. 1 Flow diagram of the study selection process. Notes:*in the authors' institutions

elaboration of an existing imaging application, 11.2\% $(32 / 286)$ concerned a new imaging application, and $4.2 \%(12 / 286)$ concerned another type of imaging as an alternative for an existing imaging application. Of the 286 studies that could directly contribute to patient care, $48.3 \%$ (138/286) would increase workload, $46.2 \%$ $(132 / 286)$ would not change workload, $4.5 \%(13 / 286)$ would decrease workload, and $1.0 \%(3 / 286)$ had an unclear effect on workload. Causes of increased workload are detailed in Fig. 2. The main cause of increased workload was an increase in interpretation time of an existing imaging application $(74.6 \%, 103 / 138)$, often combined with an increase in post-processing time and acquisition time. The second main cause of increased workload was the introduction of a completely new imaging application $(23.2 \%, 32 / 138)$.
On univariate analysis, both AI as research area and a lower impact factor of the journal in which the study was published were significantly associated with an increased workload (Table 4). On multivariate analysis, only AI as primary research area remained significantly associated with an increased workload $(p<0.001)$, with an odds ratio (OR) of 10.64 (95\% confidence interval (CI) 3.25-34.80) (Table 4). Of 38 AI studies with a potential direct contribution to patient care, 33 (86.5\%) would increase workload (of which 32 due to increased post-processing and interpretation time and 1 due to increased post-processing time), 3 (7.9\%) would decrease workload, 1 (2.6\%) would not change workload, and $1(2.6 \%)$ had an unclear effect on workload. 


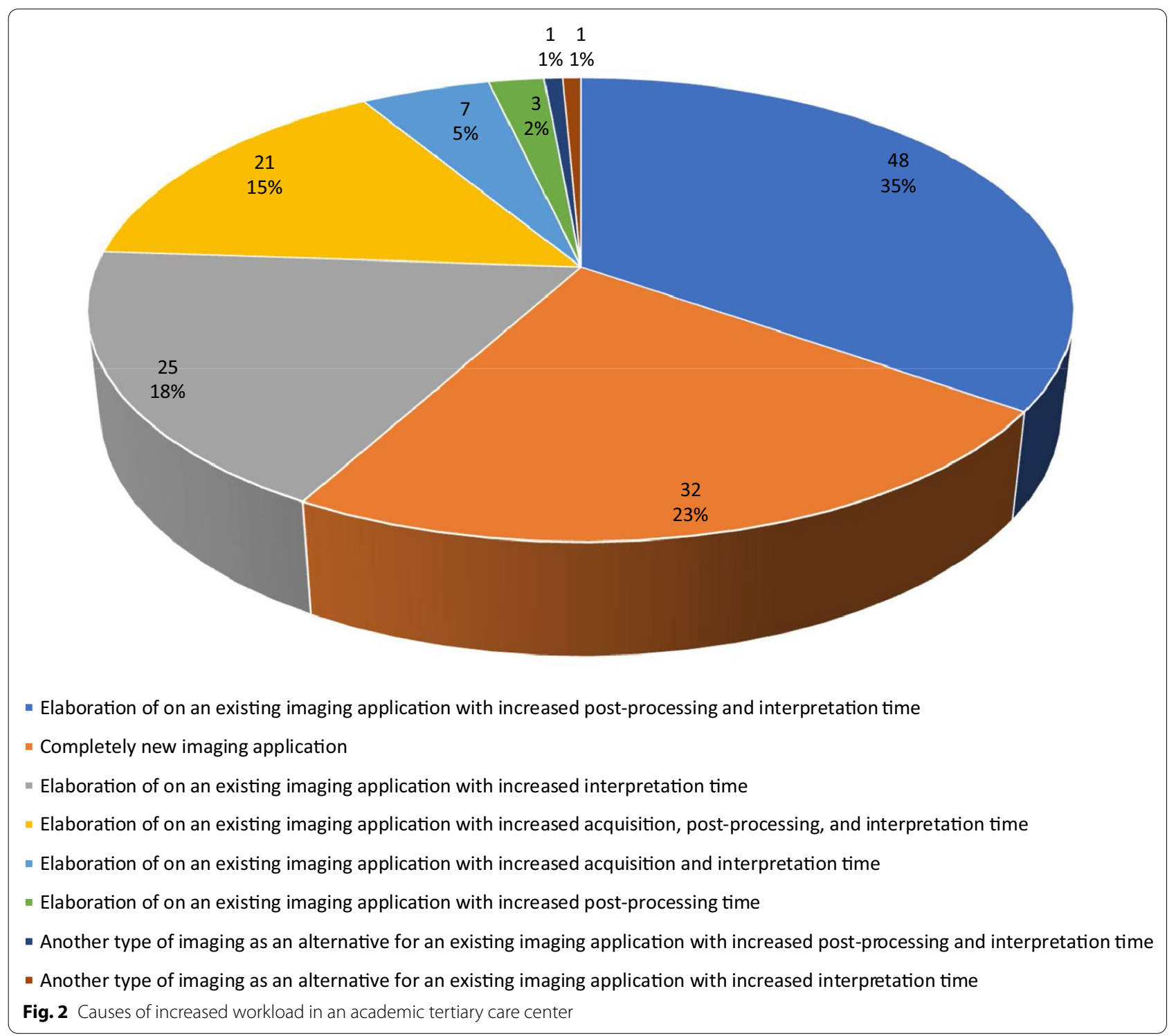

\section{Non-academic general teaching hospital setting}

$63.0 \%$ (277/440) of studies could directly contribute to patient care, of which $84.8 \%$ (235/277) concerned an elaboration of on an existing imaging application, $10.8 \%(30 / 277)$ concerned a new imaging application, and $4.3 \%(12 / 277)$ concerned another type of imaging as an alternative for an existing imaging application. Of the 277 studies that could directly contribute to patient care, 48.7\% (135/277) would increase workload, 46.2\% $(128 / 277)$ would not change workload, $4.3 \%(12 / 277)$ would decrease workload, and for $0.7 \%(2 / 277)$ the effect on workload was unclear. Causes of increased workload are detailed in Fig. 3. The main cause of increased workload was an increase in interpretation time of an existing imaging application $(74.1 \%, 100 / 135)$, often combined with an increase in post-processing time and acquisition time. The second main cause of increased workload was the introduction of a completely new imaging application $(22.2 \%, 30 / 135)$.

On both univariate and multivariate analyses, only AI as primary research area was significantly associated with an increased workload $(p<0.001)$, with a multivariate OR of 10.45 (95\% CI 3.19-34.21) (Table 5). Of 37 AI studies with a potential direct contribution to patient care, $32(86.8 \%)$ would increase workload (of which 29 due to increased post-processing and interpretation time, 2 due to increased post-processing time, and 1 due to increased interpretation time), 3 (8.1\%) would decrease workload, $1(2.7 \%)$ would not change workload, and for $1(2.7 \%)$ the effect on workload was unclear. 
Table 4 Logistic regression analyses on the association of increased workload with a study's research area and impact factor of the journal in which the study was published, for an academic tertiary care center

\begin{tabular}{|c|c|c|c|c|c|c|}
\hline \multirow[t]{2}{*}{ Variable } & \multicolumn{3}{|c|}{ Univariate analysis } & \multicolumn{3}{|c|}{ Multivariate analysis } \\
\hline & OR & $95 \% \mathrm{Cl}$ & $p$ value & OR & $95 \% \mathrm{Cl}$ & $p$ value \\
\hline $\begin{array}{l}\text { Study's research } \\
\text { area }^{\mathrm{a}}\end{array}$ & $11.79^{b}$ & $3.64-38.28^{b}$ & $<0.001^{b}$ & $10.64^{b}$ & $3.25-34.80^{b}$ & $<0.001^{\mathrm{b}}$ \\
\hline $\begin{array}{l}\text { Impact factor of } \\
\text { the journal in } \\
\text { which the study } \\
\text { was published }\end{array}$ & $0.93^{d}$ & $0.86-1.00^{d}$ & 0.020 & $0.93^{d}$ & $0.85-1.02^{\mathrm{d}}$ & 0.110 \\
\hline
\end{tabular}

$\mathrm{Cl}$ : confidence interval, OR: odds ratio

${ }^{\text {a }}$ Based on 286 studies with the following primary research areas: artificial intelligence $(n=38)$, breast $(n=16)$, cardiac ( $\left.n=32\right)$, chest $(n=20)$, computed tomography $(n=4)$, emergency $(n=1)$, gastrointestinal-abdominal $(n=21)$, head-neck $(n=6)$, magnetic resonance $(n=3)$, multisystem $(n=1)$, musculoskeletal $(n=18)$, neuroradiology $(n=46)$, nuclear medicine $(n=51)$, oncology $(n=2)$, pediatric $(n=2)$, ultrasonography $(n=2)$, urogenital $(n=17)$, and vascular $(n=6)$

${ }^{b}$ Studies with artificial intelligence as research area were significantly associated with increased workload

c Based on 26 individual journals with a median impact factor of 5.061 (range: 2.687-33.752)

d Per unit increase in impact factor

\section{Discussion}

The results of this study show that $>60 \%$ of recent scientific evidence in the medical imaging field may be directly implemented in diagnostic radiology practice for the purpose of enhancing patient care. However, nearly $50 \%$ of these studies would increase workload, while less than $5 \%$ would decrease workload. Therefore, the projected overall workload of diagnostic radiologists is expected to rise. Increased workload was due to an increase in interpretation time of an existing imaging application in nearly $75 \%$ (often combined with an increase in post-processing time and acquisition time) and due to the introduction of a completely new imaging application in $20-25 \%$. Interestingly, studies with AI as primary research area were significantly associated with an increased workload; $>86 \%$ of AI studies increased workload, of which the far majority due to an increase in both post-processing and interpretation time.

Over the past decades, the workload of radiologists has already risen considerably [1-4], with a concomitant increase in the number of studies reporting high rates of burnout among radiology staff [5, 6, 27]. Implementing recent scientific innovations and advances in knowledge in diagnostic radiology practice may benefit patient care, but, as demonstrated by the present study, further increases workload. Maintaining the same radiology workforce while increasing the intensity of work (i.e., number of procedures per time unit) and/or extending working hours has been a common phenomenon among radiology practices in the past years [28-30]. However, this strategy is not a sustainable solution considering the fact that medical imaging keeps on evolving at a rapid pace, which continuously adds to the net workload of radiologists. Without any intervention, the continuous addition of workload that aims to improve patient care will eventually turn into work overload that may jeopardize the quality and safety of patient care [7-9]. AI is regarded as a potential solution to increase efficiency and ease the workload of radiologists [11,31]. However, the present study suggests that most current AI applications in medical imaging have the opposite effect, because they commonly require additional post-processing and interpretation time rather than being seamlessly integrated in the workflow and taking over tasks of the diagnostic radiologist. Therefore, we believe that there is currently no scientific basis for policy makers to use AI as a reason to refrain from expanding the radiology workforce or to cut reimbursements for imaging procedures. To reverse the (looming) shortage of radiologists [32, 33], it may be necessary to enroll more residents into radiology training programs, train and employ radiologist assistants [34], and/or increase financial resources to employ (new) radiology staff.

The present study had some limitations. First, the results only apply to the scientific literature published in 2019. Given the lack of previous studies on this topic, the current findings can be considered as a baseline measurement to which future developments can be compared. Second, because of the lack of quantitative data on workload in almost all studies that were included in our analysis, only a qualitative analysis could be done. Third, only the direct impact of each study on workload was assessed. However, scientific innovations and advances in knowledge may allow for more reliable and accurate diagnoses, which may theoretically decrease additional imaging examinations and associated workload. Fourth, besides interpretation time, acquisition and post-processing time were 


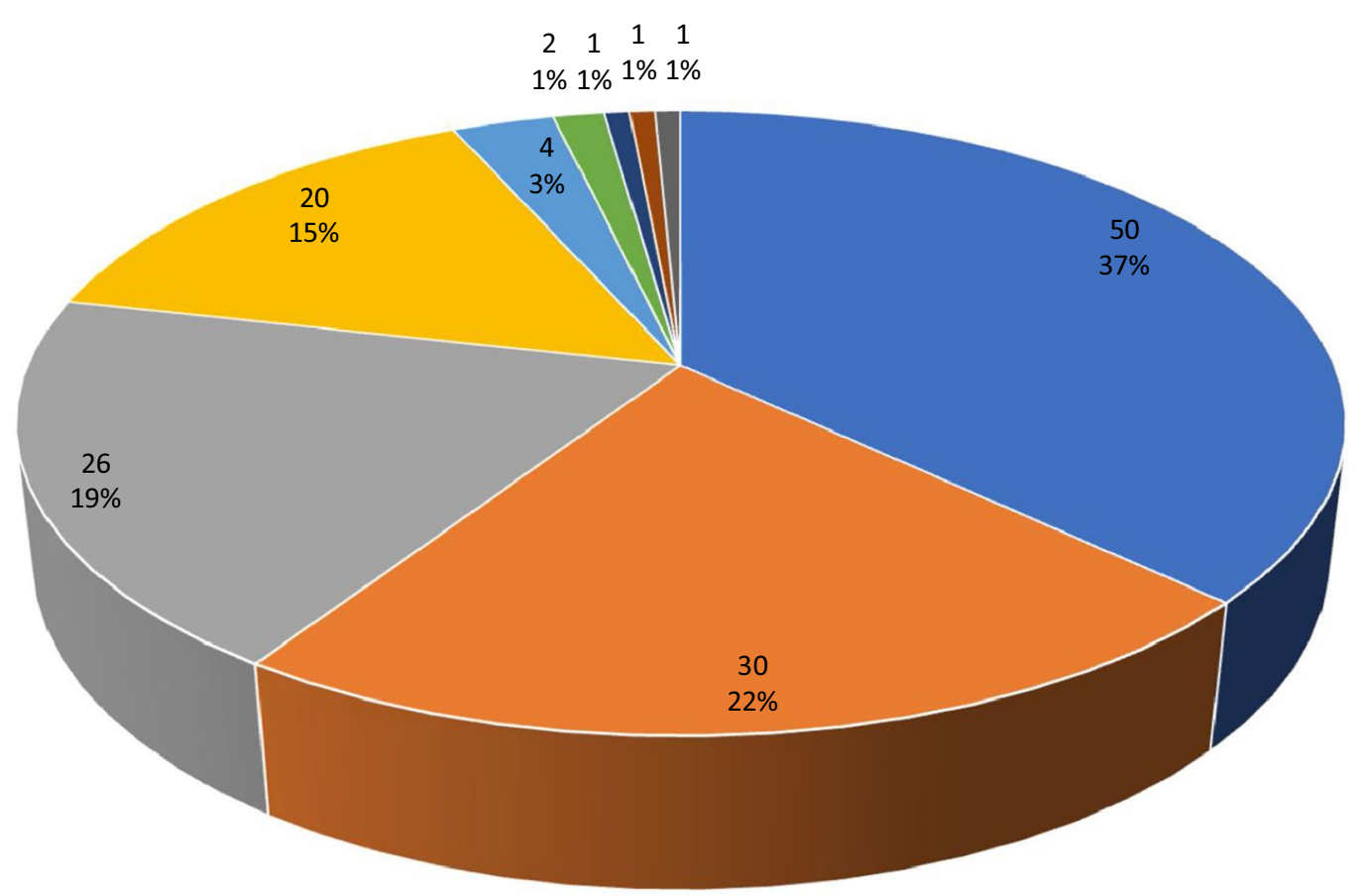

- Elaboration of on an existing imaging application with increased post-processing and interpretation time

- Completely new imaging application

- Elaboration of on an existing imaging application with increased interpretation time

- Elaboration of on an existing imaging application with increased acquisition, post-processing, and interpretation time

- Elaboration of on an existing imaging application with increased post-processing time

- Elaboration of on an existing imaging application with increased acquisition and interpretation time

- Elaboration of on an existing imaging application with increased acquisition time

- Another type of imaging as an alternative for an existing imaging application with increased post-processing and interpretation time

- Another type of imaging as an alternative for an existing imaging application with increased interpretation time

Fig. 3 Causes of increased workload in a non-academic general teaching hospital

also regarded to have a potential impact on workload, although it can be argued that they may not be the primary tasks of a radiologist. However, the radiologist bears the final responsibility for these processes, and they contribute to the overall workload of a radiology practice. Fifth, the implementation of the findings of the studies that were included in our analysis was based on a theoretical scenario. Whether or not the findings of these studies will eventually be incorporated in guidelines and experience widespread clinical implementation remains unclear. Nevertheless, the results of the present study indicate that there is no reason to assume that workload for radiologists will stabilize let alone decrease. Sixth, our findings apply to both an academic tertiary care center and a non-academic general teaching hospital in the Netherlands, as independently assessed by two radiologists. The quality of healthcare in the Netherlands ranks among the best in Europe [35]. Therefore, the results of this study are likely also applicable to modern radiology departments in other developed countries. However, further research by more radiologists, in other institutions, and in other countries, is necessary to confirm the generalizability of our results.

\section{Conclusions}

In conclusion, recently published medical imaging studies often add value to radiological patient care. However, they likely increase the overall workload of diagnostic radiologists, and this particularly applies to AI studies. 
Table 5 Logistic regression analyses on the association of increased workload with a study's research area and impact factor of the journal in which the study was published, for a non-academic general teaching hospital

\begin{tabular}{|c|c|c|c|c|c|c|}
\hline \multirow[t]{2}{*}{ Variable } & \multicolumn{3}{|c|}{ Univariate analysis } & \multicolumn{3}{|c|}{ Multivariate analysis } \\
\hline & OR & $95 \% \mathrm{Cl}$ & $p$ value & OR & $95 \% \mathrm{Cl}$ & $p$ value \\
\hline $\begin{array}{l}\text { Study's research } \\
\text { area }^{a}\end{array}$ & $11.05^{b}$ & $3.39-36.01^{b}$ & $<0.001^{b}$ & $10.45^{b}$ & $3.19-34.21^{b}$ & $<0.001^{b}$ \\
\hline $\begin{array}{l}\text { Impact factor of } \\
\text { the journal in } \\
\text { which the study } \\
\text { was published }\end{array}$ & $0.94^{d}$ & $0.87-1.01^{d}$ & 0.065 & $0.950^{d}$ & $0.87-1.04^{d}$ & 0.268 \\
\hline
\end{tabular}

$\mathrm{Cl}$ : confidence interval, OR: odds ratio

${ }^{\text {a }}$ Based on 277 studies with the following primary research areas: artificial intelligence $(n=37)$, breast $(n=16)$, cardiac ( $\left.n=32\right)$, chest ( $\left.n=18\right)$, computed tomography $(n=4)$, emergency $(n=1)$, gastrointestinal-abdominal $(n=20)$, head-neck $(n=6)$, magnetic resonance $(n=3)$, multisystem $(n=1)$, musculoskeletal $(n=18)$, neuroradiology $(n=44)$, nuclear medicine $(n=50)$, oncology $(n=1)$, pediatric $(n=2)$, ultrasonography $(n=2)$, urogenital $(n=17)$, and vascular $(n=5)$

${ }^{b}$ Studies with artificial intelligence as research area were significantly associated with increased workload

' Based on 25 individual journals with a median impact factor of 4.966 (range 2.687-33.752)

d Per unit increase in impact factor

\section{Abbreviation}

Al: Artificial intelligence; ESR: European Society of Radiology; OR: Odds ratio.

\section{Supplementary Information}

The online version contains supplementary material available at https://doi. org/10.1186/s13244-021-01031-4.

Additional file 1: Appendix 1. Search term used to retrieve studies from PubMed/Medline that were considered for inclusion. Appendix 2. Overview of 440 studies that remained after excluding 560 ineligible studies from the initial sample of 1000 studies.

\section{Authors' contributions}

TCK was involved in study concept, study design, data acquisition, data analysis and interpretation, statistical analysis, manuscript preparation, manuscript editing, and manuscript review. RMK helped in study concept, study design, data acquisition, data analysis and interpretation, statistical analysis, manuscript preparation, manuscript editing, and manuscript review. All authors have read and approved the final manuscript.

\section{Funding}

None.

\section{Availability of data and materials}

All data are available in the manuscript and attached appendices.

\section{Declarations}

\section{Ethics approval and consent to participate}

This study used literature data and did not require ethical review board approval or patient consent.

\section{Consent for publication}

Not applicable.

\section{Competing interests}

None.

\section{Author details}

${ }^{1}$ Medical Imaging Center, Departments of Radiology, Nuclear Medicine and Molecular Imaging, University Medical Center Groningen, University of Groningen, Hanzeplein 1, P.O. Box 30.001, 9700 RB Groningen, The Netherlands. ${ }^{2}$ Department of Radiology, Zuyderland Medical Center, Heerlen, Sittard-Geleen, The Netherlands.

Received: 29 April 2021 Accepted: 8 June 2021

Published online: 29 June 2021

\section{References}

1. McDonald RJ, Schwartz KM, Eckel L et al (2015) The effects of changes in utilization and technological advancements of cross-sectional imaging on radiologist workload. Acad Radiol 22:1191-1198

2. Smith-Bindman R, Kwan ML, Marlow EC et al (2019) Trends in use of medical imaging in US health care systems and in Ontario, Canada, 2000-2016. JAMA 322:843-856

3. Pola A, Corbella D, Righini A et al (2018) Computed tomography use in a large Italian region: trend analysis 2004-2014 of emergency and outpatient CT examinations in children and adults. Eur Radiol 28:2308-2318

4. Bruls RJM, Kwee RM (2020) Workload for radiologists during on-call hours: dramatic increase in the past 15 years. Insights Imaging 11:12

5. Harolds JA, Parikh JR, Bluth El, Dutton SC, Recht MP (2016) Burnout of radiologists: frequency, risk factors, and remedies: a report of the $A C R$ commission on human resources. J Am Coll Radiol 13:411-416

6. Ganeshan D, Rosenkrantz AB, Bassett RL Jr, Williams L, Lenchik L, Yang W (2020) Burnout in academic radiologists in the United States. Acad Radiol 27:1274-1281

7. Krupinski EA, Berbaum KS, Caldwell RT, Schartz KM, Kim J (2010) Long radiology workdays reduce detection and accommodation accuracy. J Am Coll Radiol 7:698-704

8. Krupinski EA, Berbaum KS, Caldwell RT, Schartz KM, Madsen MT, Kramer DJ (2012) Do long radiology workdays affect nodule detection in dynamic CT interpretation? J Am Coll Radiol 9:191-198

9. Lee CS, Nagy PG, Weaver SJ, Newman-Toker DE (2013) Cognitive and system factors contributing to diagnostic errors in radiology. AJR Am J Roentgenol 201:611-617

10. Hendee WR, Becker GJ, Borgstede JP et al (2010) Addressing overutilization in medical imaging. Radiology 257:240-245 
11. Alexander A, Jiang A, Ferreira C, Zurkiya D (2020) An intelligent future for medical imaging: a market outlook on artificial intelligence for medical imaging. J Am Coll Radiol 17:165-170

12. European Society of Radiology (ESR) (2019) Impact of artificial intelligence on radiology: a EuroAIM survey among members of the European Society of Radiology. Insights Imaging 10:105

13. 2019 Journal Citation Reports (InCites). https://jcr.clarivate.com/. Published June 23, 2020. Accessed January 8, 2021.

14. Kwaliteitsvisitatie. https://www.radiologen.nl/kwaliteit/kwaliteitsvisitatie. Publishing date unknown. Accessed January 8, 2021.

15. Mezrich R, Juluru K, Nagy P (2011) Should post-processing be performed by the radiologist? J Digit Imaging 24:378-381

16. Kronlage M, Knop KC, Schwarz D et al (2019) Amyotrophic lateral sclerosis versus multifocal motor neuropathy: utility of MR neurography. Radiology 292:149-156

17. Tanabe N, Shima H, Sato S et al (2019) Direct evaluation of peripheral airways using ultra-high-resolution CT in chronic obstructive pulmonary disease. Eur J Radiol 120:108687

18. Tsuchiya J, Yamamoto M, Bae H et al (2019) Tumor identification of less aggressive or indolent lymphoma with whole-body 11C-acetate PET/CT. Clin Nucl Med 44:276-281

19. Tagliafico AS, Cea M, Rossi F et al (2019) Differentiating diffuse from focal pattern on computed tomography in multiple myeloma: added value of a radiomics approach. Eur J Radiol 121:108739

20. Perlepe V, Michoux N, Heynen G, Vande Berg B (2019) Semi-quantitative CT assessment of fracture healing: How many and which CT reformats should be analyzed? Eur J Radiol 118:181-186

21. Matsumoto Y, Higaki T, Masuda T et al (2019) Minimizing individual variations in arterial enhancement on coronary CT angiographs using "contrast enhancement optimizer": a prospective randomized single-center study. Eur Radiol 29:2998-3005

22. Haddock B, Fan AP, Uhlrich SD et al (2019) Assessment of acute bone loading in humans using [18F]NaF PET/MRI. Eur J Nucl Med Mol Imaging 46:2452-2463

23. An H, Ma X, Pan Z, Guo H, Lee EYP (2020) Qualitative and quantitative comparison of image quality between single-shot echo-planar and interleaved multi-shot echo-planar diffusion-weighted imaging in female pelvis. Eur Radiol 30:1876-1884
24. Chen Y, Panda A, Pahwa S et al (2019) Three-dimensional MR fingerprinting for quantitative breast imaging. Radiology 290:33-40

25. Miles A, Bhatnagar G, Halligan S et al (2019) Magnetic resonance enterography, small bowel ultrasound and colonoscopy to diagnose and stage Crohn's disease: patient acceptability and perceived burden. Eur Radiol 29:1083-1093

26. Kasivisvanathan $\mathrm{V}$, Ambrosi A, Giganti F et al (2019) A dedicated prostate MRI teaching course improves the ability of the urologist to interpret clinically significant prostate cancer on multiparametric MRI. Eur Urol 75:203-204

27. Guenette JP, Smith SE (2017) Burnout: prevalence and associated factors among radiology residents in New England with comparison against United States resident physicians in other specialties. AJR Am J Roentgenol 209:136-141

28. Meghea C, Sunshine JH (2007) Determinants of radiologists' desired workloads. J Am Coll Radiol 4:166-170

29. Hillman BJ (2007) Everyone is working harder. J Am Coll Radiol 4:143-144

30. Rohatgi S, Hanna TN, Sliker CW, Abbott RM, Nicola R (2015) After-hours radiology: challenges and strategies for the radiologist. AJR Am J Roentgenol 205:956-961

31. Hosny A, Parmar C, Quackenbush J, Schwartz LH, Aerts HJWL (2018) Artificial intelligence in radiology. Nat Rev Cancer 18:500-510

32. Rimmer A (2017) Radiologist shortage leaves patient care at risk, warns royal college. BMJ 359:j4683

33. Nishie A, Kakihara D, Nojo T et al (2015) Current radiologist workload and the shortages in Japan: how many full-time radiologists are required? Jpn J Radiol 33:266-272

34. Ludwig R, Ferrara TL (2008) What is your radiologist assistant student worth to you? J Am Coll Radiol 5:115-118

35. Euro Health Consumer Index. https://healthpowerhouse.com/media/ EHCl-2018/EHCl-2018-report.pdf. Published February 25, 2020. Accessed January 8, 2021.

\section{Publisher's Note}

Springer Nature remains neutral with regard to jurisdictional claims in published maps and institutional affiliations.

\section{Submit your manuscript to a SpringerOpen ${ }^{\circ}$ journal and benefit from:}

- Convenient online submission

- Rigorous peer review

- Open access: articles freely available online

- High visibility within the field

- Retaining the copyright to your article

Submit your next manuscript at $\boldsymbol{\nabla}$ springeropen.com 